\title{
Características psicossociais de usuários de um Centro de Atenção Psicossocial CoM históRIa de Tentativa de SUICÍdio
}

\author{
Maria Ivoneide Veríssimo de Oliveira ${ }^{1}$ \\ José Gomes Bezerra Filho ${ }^{2}$ \\ Maria Vilma Neves de Lima \\ Caroline Carneiro Ferreira ${ }^{4}$ \\ Larissa Uchoa Garcia ${ }^{4}$ \\ Leirylane de Souza Pereira Goes ${ }^{5}$
}

\begin{abstract}
Objetivo: identificar características psicossociais de usuários de um Centro de Atenção Psicossocial com história de tentativa de suicídio em Fortaleza, Ceará, Brasil. Da amostra, $64,6 \%$ era do sexo feminino, $76 \%$ tinha entre $30-59$ anos, $65,5 \%$ sem companheiro. Na análise bivariada com desfecho (mais de uma tentativa de suicídio), houve associação $\mathrm{p}<0,05$ com não trabalhar, não gostar do trabalho, história de internações psiquiátricas, atendimento hospitalar, encaminhamento aos Centros de Atenção Psicossocial. Na análise multivariada, observaram-se associação com internamentos psiquiátricos como fator de risco, atendimento hospitalar como fator de proteção, além de encaminhamento. Permaneceram significativas no modelo: internações psiquiátricas como fator de risco, encaminhamento e atendimento hospitalar, como fator protetor.
\end{abstract}

Descritores: Suicídio; Tentativa de Suicídio; Fatores de Risco; Proteção Pessoal.

\footnotetext{
${ }^{1}$ MSc, Enfermeira, Centro de Ciências da Saúde, Universidade Federal do Ceará, Fortaleza, CE, Brasil.

2 PhD, Professor Adjunto, Faculdade de Medicina, Universidade Federal do Ceará, Fortaleza, CE, Brasil.

${ }^{3}$ MSc, Enfermeira, Secretaria Municipal de Saúde, Fortaleza, CE, Brasil.

${ }^{4}$ Aluna do curso de graduação em Farmácia, Universidade Federal do Ceará, Fortaleza, CE, Brasil.

${ }^{5}$ Mestranda, Universidade Federal do Ceará, Fortaleza, CE, Brasil.
} 


\title{
Psychosocial CHaRacteristics of Patients With A History OF SUICIDE attempts at a Center for Psychosocial Care (CAPS)
}

\begin{abstract}
Objective - To identify the psychosocial characteristics of patients with a history of suicide attempts at a CAPS Fortaleza, Ceará, Brazil. Of the sample, $64.6 \%$ were female, $76 \%$ with ages varying from $30-59$ and $65.5 \%$ were unmarried. The bivariate analysis with outcome (more than one suicide attempt), showed an association of $p<0.05$ with not working, does not like the work, history of psychiatric confinement, hospital care, referred to the CAPS. In the multivariate analysis, the association with psychiatric confinement was identified as a risk factor, the hospital care as a protective factor, as well as the referral. The significant factors remained the same in the model: psychiatric confinement as a risk factor, referral and hospital care, as a protective factor.
\end{abstract}

Descriptors: Suicide; Suicide, Attempted; Risk Factors; Personal Protection.

\section{Características PSicosociales de usuarios de UN CAPS CON HISTORIA DE TENTATIVA DE SUICIDIO}

Objetivo-Identificar características psicosociales de usuarios de un CAPS con historia de tentativa de suicidio. Fortaleza-Ceará-Brasil. De la muestra, 64,6\% eran del sexo femenino, 76\% entre 30-59 años, 65,5\% sin compañero. En el análisis bivariado con final (más de una tentativa de suicidio), hubo asociación $\mathrm{p}<0,05$ con no trabajar, no le gustar el trabajo, historia de internaciones psiquiátricas, servicio hospitalario, encaminamiento a los CAPS. En el análisis multivariado, asociación con internamientos psiquiátricos como factor de riesgo, servicio hospitalario como factor de protección, como también encaminamiento. Permanecieron significativas en el modelo: internaciones psiquiátricas como factor de riesgo, encaminamiento y servicio hospitalario, como factor protector.

Descriptores: Suicídio; Intento de Suicidio; Factores de Riesgo; Protección Personal.

\section{Introdução}

Por suicídio entende-se a ação de tirar a própria vida. Em obra clássica, no século XIX (1898), Émile Durkheim definiu o suicídio como caso de morte que resulte, direta ou indiretamente, de ato positivo ou negativo, praticado pela vítima, com conhecimento do resultado ${ }^{(1)}$. Classifica esse ato como fenômeno social e o subdivide em quatro tipos: egoísta, altruísta, anômico e fatalista. Enquanto o egoísta é o resultado de individuação excessiva, com ênfase na autonomia da consciência individual e na ausência de laços familiares ou de interações sociais significativas, o anômico associa-se ao desregramento, crises e mudanças pelo enfraquecimento da malha social, e o altruísta explica-se pela subordinação do indivíduo a fins sociais, ato percebido como dever, gesto impessoal. Quanto ao fatalista, embora fosse visto como de pouca relevância contemporânea, na ótica do autor isso acontecia quando um indivíduo era regulado demais pela sociedade. Nesse cenário, a opressão do indivíduo resultava em um sentimento de impotência diante do destino ou da sociedasde ${ }^{(2)}$.

Sobre sua etiologia, de forma geral, há destaque para alguns fatores de risco, tais como: os psicossociais, as doenças crônicas, a biologia, a personalidade, os transtornos psiquiátricos e a história genética e familiar como de extrema importância ${ }^{(3)}$. 
Hoje o suicídio é considerado problema de saúde pública em muitos países, principalmente no Brasil, por ser um país em desenvolvimento. Afeta todas as classes sociais e tem aumentado dramaticamente nos últimos anos, apesar do esforço na prevenção ${ }^{(4)}$.

De acordo com determinados autores, o comportamento suicida é classificado em três diferentes categorias ou domínios: ideação suicida, tentativa de suicídio e suicídio consumado ${ }^{(3)}$. O comportamento suicida é visto como "todo ato pelo qual um indivíduo causa lesão a si mesmo, qualquer que seja o grau de intenção letal e de conhecimento do verdadeiro motivo desse ato". Essa noção concebe comportamento suicida com ideia de autodestruição, e passa de ameaça e gesto a tentativa e, finalmente, consuma-se o ato suicida ${ }^{(5)}$. Ideação suicida se refere a pensamentos de autodestruição ou a ideias suicidas. Engloba desejos, atitudes ou planos do indivíduo de pôr fim à própria vida. Provavelmente a identificação precoce das ideias ajuda a evitar tentativas e a prevenir autodano exitoso ${ }^{(3)}$. No plano de suicídio, o indivíduo está decidido a dar cabo à própria vida, trama a morte e planeja detalhes como método, local e horário ${ }^{(6)}$. Além dos fatores de risco mencionados, determinados autores sugerem que pacientes com transtorno psiquiátrico têm mais de $50 \%$ de chances de chegar ao suicídio ${ }^{(7)}$.

Em estudo pertinente, os autores referem, entre os que tentam o suicídio, que de 10 a $15 \%$ completam o ato $^{(8)}$. Depressão e doenças físicas foram os principais fatores de risco associados à tentativa de suicídio em $\operatorname{idosos}^{(9)}$. Embora seja impossível prever suicídios, as taxas na população em geral podem ser reduzidas por meio de assistência adequada feita por profissionais com habilidades na identificação e tratamento dos fatores de $\operatorname{risco}^{(8)}$.

Em âmbito mundial, o coeficiente de mortalidade por suicídio é de 16 casos/100.000 habitantes ${ }^{(1)}$. No Brasil esse coeficiente é, em média, de 4,5 por 100.000 habitantes, portanto, relativamente baixo em escala mundial. Contudo, em cidades e regiões, bem como em grupos populacionais (por exemplo, de jovens em grandes cidades, de indígenas do Centro-Oeste e do Norte, e entre lavradores do interior do Rio Grande do Sul), as cifras se aproximam ou superam países com taxas elevadas, como do Leste Europeu e da Escandinávia. Como divulgado, os coeficientes de suicídio têm aumentado no país, notadamente entre jovens e adultos jovens do sexo masculino ${ }^{(6)}$.

No Ceará, em 2004, a mortalidade por suicídio representou $9,4 \%$ de óbitos registrados, com maior concentração no sexo masculino ${ }^{(10)}$. Em Fortaleza, de 1998 a 2008, ocorreram 1.299 óbitos por suicídio, 1.040 do sexo masculino e 259, do feminino. Entre homens, a mortalidade foi de 9,9 por 100.000 habitantes, entre mulheres, 2,2 por 100.000 habitantes ${ }^{(10)}$.

No caso de tentativa de suicídio, o panorama é mais grave. Conforme se aponta, o número pode ser até quarenta vezes maior que o de suicídios. A Organização Mundial de Saúde (OMS), em perspectiva mundial, indica valor superior em até vinte vezes. Na população jovem, de 15 a 44 anos, as lesões ou traumas decorrentes de tentativa de suicídio representam a sexta maior causa de problemas de saúde e incapacitação física. Pelos dados, essa tentativa representa grave problema de saúde pública mundial, que atinge cada vez mais jovens, com sérios danos à saúde, incluindo psicológicos e socioeconômicos ${ }^{(11)}$. As tentativas de suicídio exercem considerável impacto nos serviços de saúde e, de acordo com estimativas, 1,4\% da carga global de doenças, no ano 2002, deveu-se às tentativas de suicídio. Essa cifra deverá chegar aos 2,4\%, em $2020^{(6)}$. Ainda, como a OMS aponta, há uma morte por suicídio a cada quarenta segundos e uma tentativa a cada três. Para cada suicídio, há pelo menos dez tentativas suficientemente sérias a ponto de exigir atenção médica; e para cada tentativa registrada há quatro desconhecidas. $\mathrm{O}$ suicídio tem visível impacto em pelo menos seis pessoas, dentro da família, amigos e outros; o impacto psicológico, social e financeiro é imensurável ${ }^{(5)}$. Diante da escassez de dados sobre o tema em Fortaleza, decidiu-se desenvolver a pesquisa, a qual constitui parte de uma tese de doutorado, cujo objetivo foi descrever as principais características de vítimas de tentativas de suicídio em instituições públicas dessa cidade, no período de junho de 2011 a maio de 2012. Com base na literatura nacional e internacional, realizouse o estudo com o objetivo de identificar as características psicossociais de usuários de um Centro de Atenção Psicossocial (CAPS) com história de tentativa de suicídio.

\section{Metodologia}

Estudo transversal, quantitativo, descritivo e analítico, desenvolvido no Centro de Atenção Psicossocial em Fortaleza, capital do Estado do Ceará, Brasil, com atendimento a pacientes referenciados de outros serviços de saúde, como urgência psiquiátrica ou egressos de internação hospitalar. O mencionado centro é um serviço da Regional III da Secretaria de Saúde do município e abrange bairros da região metropolitana de Fortaleza.

Adotou-se amostra por conveniência, composta por todos os atendimentos com diagnóstico de tentativa de suicídio, prestados no CAPS. A coleta de dados ocorreu durante doze meses consecutivos, em plantões de doze horas, três vezes por semana, conforme atendimento psiquiátrico, entre junho de 2011 e maio de 2012. Mediante visita ao CAPS, inicialmente analisaram-se todos os prontuários de pacientes agendados para atendimento médico após tentativa de suicídio. Feita a identificação, no momento da consulta, o paciente era convidado a participar do estudo.

Os dados foram coletados por meio de entrevista com duração média de trinta minutos, seguindo um formulário padronizado, aplicado por acadêmicos de Enfermagem, Farmácia, além de outros profissionais previamente capacitados e supervisionados por técnicos do Grupo de Violência da Faculdade de Medicina/Universidade Federal do Ceará. Integraram a amostra 113 pacientes identificados com tentativas de suicídio. Incluiu-se na pesquisa toda vítima atendida com tentativa de suicídio, pelo menos uma vez, em qualquer período da vida. Não houve exclusão. Como inicialmente analisava-se o prontuário, aqueles com 
dúvida de diagnóstico de tentativa de suicídio e incapazes de responderem aos instrumentos não eram selecionados. Investigaram-se as seguintes variáveis: sexo, idade, nível de escolaridade, estado civil, renda familiar, ter trabalho, religião, gostar do trabalho, ter amigos, internações psiquiátricas, tipo de atendimento e encaminhamento.

Utilizou-se a décima revisão da Classificação Internacional de Doenças (CID-10), compreendendo as categorias x60 a x84. No primeiro momento, os dados foram analisados com auxílio do programa Stata, versão 10, por meio de estatística descritiva, pela elaboração de frequências absolutas e percentuais; em seguida, foram organizados em tabelas e estudados com base na literatura pertinente. Executou-se análise bivariada entre a variável desfecho (no caso de mais de uma tentativa de suicídio) e os fatores predisponentes em associação, por meio do teste exato de Fischer com $\mathrm{p}<0,050$. Na regressão logística multivariada foram incluídas no modelo final variáveis com significância estatística $\mathrm{p} \leq 0,20$.

Em consonância com o exigido, a pesquisa foi submetida ao Comitê de Ética em Pesquisa, da Maternidade-Escola Assis Chateaubriand/Universidade Federal do Ceará, conforme Resolução nº196, de 10 de outubro de 1996, do Conselho Nacional de Saúde, tendo sido aprovada por ele.

\section{Resultados}

$\mathrm{Na}$ Tabela 1, expõem-se as caracterísitcas sociodemográficas dos participantes. Das 113 vítimas pesquisadas, $66,4 \%$ haviam tentado o suicídio mais de uma vez ao longo da vida. Desses, $64,6 \%$ eram do sexo feminino, ou seja, mais da metade da amostra. Em relação à faixa etária, $76,0 \%$ deles estavam entre 30 e 59 anos. De acordo com os dados, havia $75,2 \%$ sem nenhuma escolaridade ou cursado até o ensino fundamental. No referente ao estado civil, a maior proporção de tentativas ocorreu entre os pacientes sem vínculos afetivos (65,5\%). Quanto à renda, poderá ser considerada baixa, uma vez que a maioria $(85,8 \%)$ recebia até um salário-mínimo. Ademais, a minoria trabalhava $(16 ; 14,0 \%)$, e entre esses, $68,7 \%$ eram motivados em fazê-lo. No tocante ao convívio social, 67,0\% responderam ter amigos. Sobre religião, observou-se predominância entre eles com filiação religiosa $(96,5 \%)$. No entanto, a maioria $(65,0 \%)$ quase nunca frequentava a igreja ou algum culto. Ao se tomar a amostra como um todo, 68,0\% tinham história de internações em hospitais psiquiátricos. $\mathrm{O}$ atendimento hospitalar, após tentativa de suicídio, foi maior $(79,0 \%)$ quando comparado ao verificado em posto de saúde. Do total, $61,0 \%$ foram encaminhados para os CAPSs.

Tabela 1 - Distribuição dos entrevistados, segundo as características sociodemográficas. Fortaleza, CE, Brasil, 2012

\begin{tabular}{|c|c|c|}
\hline Características & Frequência & $\%$ \\
\hline \multicolumn{3}{|l|}{ Número de tentativas de suicídio } \\
\hline $1 \mathrm{vez}$ & 38 & 33,6 \\
\hline$>1 \mathrm{vez}$ & 75 & 66,4 \\
\hline \multicolumn{3}{|l|}{ Sexo } \\
\hline Masculino & 40 & 35,4 \\
\hline Feminino & 73 & 64,6 \\
\hline \multicolumn{3}{|l|}{ Idade } \\
\hline $14-29$ anos de idade & 26 & 23,0 \\
\hline 30-59 & 85 & 76,0 \\
\hline 60 anos ou mais & 2 & 1,0 \\
\hline \multicolumn{3}{|l|}{ Escolaridade } \\
\hline Nenhuma ou cursado até o ensino fundamental & 85 & 75,2 \\
\hline Ensino médio e superior & 28 & 24,8 \\
\hline \multicolumn{3}{|l|}{ Estado civil } \\
\hline Sem vínculo afetivo (solteiro, separado, divorciado e viúvo) & 74 & 65,5 \\
\hline Com vínculo afetivo (casado e mora junto) & 39 & 34,5 \\
\hline \multicolumn{3}{|l|}{ Renda familiar } \\
\hline Até um salário-mínimo & 97 & 85,8 \\
\hline Mais de um salário-mínimo & 16 & 14,2 \\
\hline \multicolumn{3}{|l|}{ Trabalha } \\
\hline Sim & 16 & 14,0 \\
\hline Não & 97 & 86,0 \\
\hline \multicolumn{3}{|l|}{ Gostar do trabalho } \\
\hline Sim & 11 & 68,7 \\
\hline Não & 5 & 31,3 \\
\hline \multicolumn{3}{|l|}{ Ter amigos } \\
\hline Sim & 76 & 67,0 \\
\hline Não & 37 & 33,0 \\
\hline
\end{tabular}


Table 1 - (continuation)

\begin{tabular}{lcc}
\hline \multicolumn{1}{c}{ Características } & Frequência & $\%$ \\
\hline Religião & & 3,5 \\
Sem filiação religiosa & 4 & 96,5 \\
Com filiação religiosa & 109 & 35,0 \\
Frequência com que vai à igreja ou ao culto & & 65,0 \\
Sempre & 40 & 68,0 \\
Quase nunca & 73 & 32,0 \\
Internações psiquiátricas & 77 & 79,0 \\
Sim & 36 & 21,0 \\
Não & & \\
Atendimento & 90 & 61,0 \\
Hospitalar & 23 & 39,0 \\
Posto de saúde & & \\
Encaminhamento & 69 & 43 \\
CAPS & 43 & \\
Outros
\end{tabular}

Fonte: CAPS GERAL III. Fortaleza, Ceará, 2012

$\mathrm{Na}$ Tabela 2, consta a análse bivariada entre o desfecho (mais de uma tentativa de suicídio) e variáveis independentes. Como observado, de início, as variáveis sexo, idade, estado civil, escolaridade, renda familiar, religião, frequência à igreja ou ao templo, amigos não apresentam associação estatística $(\mathrm{p}>0,05)$.

Não trabalhar mostrou associação com mais de uma tentativa de suicídio, $\mathrm{RC}=3,01$ (IC95\%:1,02-8,87) p=0,045. Vítimas que relataram não gostar de trabalhar denotaram risco treze vezes maior de tentativa de suicídio do que aquelas que relataram gostar, $\mathrm{RC}=13,3$ (IC95\%:1,06-166) $\mathrm{p}=0,044$. Da mesma forma, vítima com história de internações psiquiátricas revelou-se como variável significativamente associada ao desfecho estudado, RC=4,09 (IC95\%: 1,769,51), $\mathrm{p}=0,001$. Quanto ao tipo de atendimento após tentativa, o hospitalar funcionou como fator de proteção, $\mathrm{RC}=0,23$ (IC95\%: 0,08-0,60), $\mathrm{p}=0,001$. No tocante aos encaminhamentos dos entrevistados, relacionados ao desfecho, também se mostrou associado como fator de proteção, RC=0,21 (IC95\%: 0,09-0,48), $\mathrm{p}=0,001$.

Tabela 2 - Regressão logística bivariada com o desfecho (mais de uma tentativa de suicídio) e variáveis independentes. Fortaleza, CE, Brasil, 2012

\begin{tabular}{|c|c|c|c|c|c|c|c|}
\hline \multirow{2}{*}{ Características } & \multicolumn{2}{|c|}{$\begin{array}{c}\text { Tentou suicídio mais } \\
\text { de uma vez }\end{array}$} & \multicolumn{2}{|c|}{$\begin{array}{c}\text { Tentou suicídio uma } \\
\text { vez }\end{array}$} & \multirow{2}{*}{$\begin{array}{l}\text { Razão } \\
\text { chances }\end{array}$} & \multirow{2}{*}{ IC95\% } & \multirow{2}{*}{$\mathrm{p}$} \\
\hline & $\mathrm{n}$ & $\%$ & $n$ & $\%$ & & & \\
\hline Sexo & & & & & 0,96 & $0,42-2,19$ & 0,928 \\
\hline Feminino & 48 & 64,0 & 24 & 65,0 & & & \\
\hline Masculino & 27 & 36,0 & 13 & 35,0 & & & \\
\hline Idade & & & & & 0,60 & $0,23-1,56$ & 0,303 \\
\hline$<19$ & 19 & 25,0 & 7 & 19,0 & & & \\
\hline $30-59$ & 56 & 75,0 & 29 & 78,0 & & & \\
\hline$>60$ & - & - & 1 & 3,0 & & & \\
\hline Estado civil & & & & & 1,16 & $0,51-2,63$ & 0,711 \\
\hline Com vínculo & 25 & 33,0 & 14 & 37,0 & & & \\
\hline Sem vínculo & 50 & 67,0 & 24 & 63,0 & & & \\
\hline Escolaridade & & & & & 1,38 & $0,57-3,36$ & 0,466 \\
\hline Até o ensino fundamental & 58 & 77,0 & 27 & 71,0 & & & \\
\hline Ensino médio e superior & 17 & 23,0 & 11 & 29,0 & & & \\
\hline Renda familiar & & & & & 0,61 & $0,18-2,06$ & 0,434 \\
\hline Até um salário & 63 & 84,0 & 34 & 89,0 & & & \\
\hline Mais de um salário & 12 & 16,0 & 4 & 11,0 & & & \\
\hline Filiação religiosa & & & & & 1,54 & $0,15-15,3$ & 0,712 \\
\hline Sim & 3 & 4,0 & 1 & 3,0 & & & \\
\hline Não & 72 & 96,0 & 37 & 97,0 & & & \\
\hline Frequência com que vai à igreja ou ao culto & & & & & 1,83 & $0,81-4,09$ & 0,142 \\
\hline Sempre & 23 & 31,0 & 17 & 45,0 & & & \\
\hline Quase nunca & 52 & 69,0 & 21 & 55,0 & & & \\
\hline
\end{tabular}


Table 2 - (continuation)

\begin{tabular}{|c|c|c|c|c|c|c|c|}
\hline \multirow{2}{*}{ Características } & \multicolumn{2}{|c|}{$\begin{array}{c}\text { Tentou suicídio mais } \\
\text { de uma vez }\end{array}$} & \multicolumn{2}{|c|}{$\begin{array}{c}\text { Tentou suicídio uma } \\
\text { vez }\end{array}$} & \multirow{2}{*}{$\begin{array}{l}\text { Razão } \\
\text { chances }\end{array}$} & \multirow{2}{*}{ IC95\% } & \multirow{2}{*}{$\mathbf{p}$} \\
\hline & $\mathbf{n}$ & $\%$ & $\mathbf{n}$ & $\%$ & & & \\
\hline Trabalha & & & & & 3,01 & $1,02-8,87$ & 0,045 \\
\hline Sim & 7 & 10,0 & 9 & 24,0 & & & \\
\hline Não & 68 & 90,0 & 29 & 76,0 & & & \\
\hline Gostar do trabalho & & & & & 13,30 & $1,06-166$ & 0,044 \\
\hline Sim & 3 & 43,0 & 8 & 89,0 & & & \\
\hline Não & 4 & 57,0 & 1 & 11,0 & & & \\
\hline Ter amigos & & & & & 0,90 & $0,39-2,06$ & 0,813 \\
\hline Sim & 51 & 68,0 & 25 & 66,0 & & & \\
\hline Não & 24 & 32,0 & 13 & 34,0 & & & \\
\hline Internação anterior & & & & & 4,09 & $1,76-9,51$ & 0,001 \\
\hline Sim & 59 & 79,0 & 18 & 47,0 & & & \\
\hline Não & 16 & 21,0 & 20 & 53,0 & & & \\
\hline Atendimento & & & & & 0,23 & $0,08-0,60$ & 0,003 \\
\hline Hospitalar & 66 & 88,0 & 24 & 63,0 & & & \\
\hline Posto de saúde & 9 & 12,0 & 14 & 34,0 & & & \\
\hline Encaminhamento & & & & & 0,21 & $0,09-0,48$ & 0,001 \\
\hline CAPS & 55 & 73,0 & 14 & 37,0 & & & \\
\hline Outros & 20 & 27,0 & 24 & 63,0 & & & \\
\hline
\end{tabular}

Fonte: CAPS GERAL III. Fortaleza, Ceará, 2012; p=teste exato de Fisher; IC95\%= Intervalo de Confiança.

$\mathrm{Na}$ análise de regressão logística multivariada foram incluídas variáveis em associação significativa com desfecho (mais de uma tentativa de suicídio) com $\mathrm{p}<0,20$.

De acordo com a Tabela 3, na análise de regressão logística multivariada, após controle de fatores de confusão com as variáveis independentes, o risco de mais de uma tentativa de suicídio manteve associação estatisticamente significativa com: história de internamento psiquiátrico com mais de uma tentativa de suicídio, OR=3,55 (IC95\% $1,44-8,75) \mathrm{p}=0,006$; atendimento hospitalar após tentativa de suicídio tem prevalência significativamente como fator de proteção, $\mathrm{OR}=0,30$ (IC95\% 0,10-0,86) $\mathrm{p}=0,026$, como também encaminhamento para os CAPSs, OR=0,56 (IC95\% 0,32-0,97) $\mathrm{p}=0,040$.

Tabela 3 - Análise de regressão logística multivariada com o desfecho (mais de uma tentativa de suicídio) e variáveis independentes. Fortaleza, CE, Brasil,, 2012

\begin{tabular}{|c|c|c|c|c|c|c|}
\hline Relação & Odds bruto & IC95\% & p & Odds ajustado & IC95\% & p \\
\hline Internamento anterior em hospital psiquiático & 4,09 & $1,76-9,51$ & 0,001 & 3,55 & $1,44-8,75$ & 0,006 \\
\hline Atendimento hospitalar & 0,23 & $0,00-0,03$ & 0,000 & 0,30 & $0,10-0,86$ & 0,026 \\
\hline Encaminhamento para o CAPS & 0,21 & $0,09-0,48$ & 0.000 & 0,56 & $0,32-0,97$ & 0,040 \\
\hline
\end{tabular}

p=teste exato de Fisher; IC95\%=Intervalo de Confiança.

\section{Discussão}

Foram abordadas 113 vítimas. Dessas, 66,4\% fizeram mais de uma tentativa de suicídio. Esse dado é preocupante, pois a literatura mostra aumento de risco de suicídio de pessoas com histórico de tentativas anteriores ${ }^{(12)}$.

Como verificado, 64,6\% $(n=73)$ das tentativas de suicídio foram do sexo feminino, portanto, corroborando a literatura ${ }^{(3-13)}$. Conforme estudo em Ribeirão Preto, SP, $77,8 \%$ dos casos pertencem ao sexo feminino ${ }^{(14)}$. No entanto, em estudo realizado em Minas Gerais, entre 1998 e 2003, de 14.443 internações no SUS, 55,4\% eram do sexo masculino $^{(5)}$. Quanto à faixa etária, 76,0\% estavam entre 30 e 59 anos de idade. Em Minas Gerais, identificaramse dois picos de taxa de suicídio entre jovens (de 10 a 14 anos e de 15 a 19 anos) ${ }^{(5)}$. Em hospital de emergência em
Fortaleza, em 2000, indicaram-se também dois picos: 35\% entre 21 e 30 anos e $32 \%$ entre 10 e $20^{(13)}$.

Tem-se, ainda, que $75,2 \%$ dos pacientes são apresentados como sem nenhuma escolaridade ou cursaram apenas até o ensino fundamental. Esses dados demonstram baixa escolaridade. A pesquisa corrobora os achados de estudo realizado em Itabira (Minas Gerais), no qual se evidenciou que as principais vítimas de suicídios são pessoas que têm apenas o ensino fundamental. Consoante os autores referem, o fato decorre possivelmente da falta de oportunidades no meio social e econômico ${ }^{(15)}$. Também, em hospital, no Rio de Janeiro, verifica-se baixo nível educacional $(66,7 \%)^{(8)}$.

Neste estudo, quanto ao estado civil, há predomínio de pacientes sem vínculo afetivo $(65,5 \%)$, achados de acordo com os encontrados sobre análise epidemiológica de 
suicídio no Brasil, entre 1980 e $2006^{(12)}$, e em Mato Grosso do $\mathrm{Sul}^{(16)}$. Como consta em estudos epidemiológicos, em geral, a taxa de suicídio é maior entre solteiros, viúvos e divorciados que entre casados $^{(17)}$. Das vítimas, 97 $(85,8 \%)$ mencionaram renda familiar de até um saláriomínimo, assim, mais propensos à tentativa de suicídio pelas dificuldades da vida socioeconômica. Tais dados corroboram estudo sobre tentativa e óbitos por suicídio no Ceará, em que a maioria tem uma renda inferior a um salário-mínimo ${ }^{(18)}$.

Ainda, como observado no estudo ora mencionado, proporção pequena de pessoas trabalhavam (14,0\%); no entanto, a maioria $(68,7 \%)$ gostava de fazê-lo. Autores apontam, em pesquisa, que trabalhar está associado com melhor saúde e menos riscos de mortalidade ${ }^{(19)}$. Quanto à convivência, a maioria $(67,0 \%)$ referiu ter amigos. Sobre planejamento suicida, segundo estudo, boas relações com amigos e colegas representam um dado de extrema importância como fator de proteção para a qualidade de vida $^{(3)}$.

Destacou-se a predominância de pacientes com filiação religiosa $(96,5 \%)$, embora a maior parte $(65,0 \%)$ tenha respondido quase nunca frequentar a igreja ou algum culto. Corrobora-se, portanto, estudo realizado no Rio Grande do Sul, segundo o qual, entre os fatores protetores contra tentativa de suicídio, está a religião ${ }^{(3)}$.

$\mathrm{Na}$ amostra, a variável história de internamento em hospital psiquiátrico representou $68,0 \%$. Sobre prevenção do comportamento suicida, a maioria das vítimas sofre de algum transtorno psiquiátrico, necessitando muitas vezes de internamento ${ }^{(20)}$. Nessa ótica, o atendimento hospitalar após tentativa foi importante $(79,0 \%)$. De acordo com a literatura, pacientes com comportamentos suicidas geralmente têm doença mental diagnosticada, o que aumenta o risco de tentativa de suicídio, com agressões graves e, muitas vezes, com necessidade de atendimento hospitalar ${ }^{(21)}$.

Por fim, encaminhamentos para tratamento nos CAPSs chegaram a $61,0 \%$ da amostra estudada. O atendimento em serviços de saúde especializados parece ser a forma mais efetiva de prevenção. Consoante estudo realizado em Gotland, Suécia, entre pacientes em acompanhamento médico, houve diminuição do coeficiente de suicídio ${ }^{(22)}$.

Na análise bivariada, a variável não trabalhar prevalece sobre tentativas de suicídio entre os que trabalham. Tal achado é corroborado por estudo sobre características epidemiológicas do suicídio no Rio Grande do Sul, no qual os autores encontraram associação estatística entre desemprego e suicídio, podendo o paciente ser também afetado pela mortalidade ${ }^{(23)}$. Ademais, os participantes que afirmaram não gostar de trabalhar apresentaram um risco de treze vezes para mais de uma tentativa de suicídio do que os demais. Desemprego é fator de risco. Emprego e satisfação com a vida, capacidade de adaptação positiva são fatores de proteção ${ }^{(24)}$.

Entre variáveis em associação estatística com desfecho, fato interessante a ser observado foi a significância estatística entre mais de uma tentativa de suicídio e história de internação em hospital psiquiátrico.
Identificaram-se 79,0\% com história de internamento anterior com mais de uma tentativa e risco de quatro vezes mais. No entanto, em estudo desenvolvido em São Paulo, não foi encontrada associação entre tentativa de suicídio e internações psiquiátricas ${ }^{(25)}$.

Relação entre atendimento hospitalar e número de tentativas de suicídio esteve fortemente associada como fator de proteção, quando se comparam pacientes que tiveram esse atendimento aos sem atendimento. Destacase a importância do sistema na captação dos casos, com possibilidade de coleta de informações significativas para, no futuro, subsidiar planejamento de políticas, prevenção e proteção efetivas, para redução do agravo na população.

Evidentemente, o acompanhamento em unidades de saúde como CAPS é fundamental, com significância estatística marcante no desfecho, como fator de proteção. $\mathrm{O}$ atendimento é uma experiência em saúde mental em que se observa, analisa e se tenta dar qualidade de vida às pessoas. $\mathrm{Na}$ ótica de determinados autores, a melhor estratégia para as populações de alto risco é melhorar os serviços de saúde e desenvolver intervenções efetivas em pacientes com tentativa de suicídio, por meio de acompanhamento adequado ${ }^{(24)}$. Nesse âmbito, o conhecimento do perfil epidemiológico é um passo para alertar acerca do papel da prevenção de suicídio, assim como abrir caminho para a capacitação dos profissionais de saúde com foco no atendimento, em específico, para identificação de risco suicida, assim como implementação de possível intervenção.

Ao longo do estudo, verificaram-se algumas limitações metodológicas. No CAPS, durante o período da coleta de dados, os atendimentos se reduziram em virtude da queda no número de psiquiatras na instituição, diminuindo-se as vítimas atendidas e entrevistadas.

Ressalta-se, ainda: a amostra não foi representativa de todos os casos de tentativa de suicídio no município de Fortaleza. Constaram-se apenas as que buscaram atendimento médico no CAPS da Regional III, da Secretaria de Saúde. Não se sabe, pois, como se comportam os que tentam o suicídio e chegam ao atendimento médico particular.

\section{Conclusão}

No referente aos resultados, determinados pontos merecem considerações: propensão da mulher à tentativa de suicídio mais que do homem, baixa escolaridade, sem vínculos afetivos e com baixa renda. Tem-se que vítimas com história de internamento psiquiátrico apresentam risco mais elevado em comparação com os sem história. Como mostra a pesquisa, é necessário aprofundar a avaliação dos dados, em especial para adoção de medidas de prevenção e intervenção. Os dados não são tão alarmantes quando comparados aos de outros estados e capitais brasileiras, contudo, atente-se para a importância da prevenção, assim como para a capacitação dos profissionais de saúde para atendimento, no intuito de minimizar a situação.

Conhecer o perfil de pessoas que tentam suicídio é essencial para a equipe de saúde, porquanto possibilita 
melhor atendimento e, talvez, um repensar da vontade de concretizar o ato. Por ser o suicídio considerado problema de saúde pública mundial, gestores, profissionais de saúde, na identificação de pessoas com baixa autoestima, sem perspectiva para o futuro, utilizam estratégias educativas de valorização da vida, resgate da autoestima e de perspectivas da população vulnerável à prática suicida. Diante da situação, é fundamental que os serviços de saúde sejam devidamente preparados e estruturados para atendimento às vítimas, em articulação com redes de apoio no acompanhamento dessas pessoas. Espera-se que este trabalho possa contribuir para o alcance desses objetivos e para a implementação de futuros estudos, com vistas à prevenção e intervenção na vida de sujeitos expostos a atos dessa natureza.

\section{Referências}

1. Parente ACM, Soares RB, Araújo ARF, Monteiro CFS. Caracterização dos casos de suicídio em uma capital do Nordeste Brasileiro. Rev Bras Enferm. julho-ago 2007;60(4):377-81.

2. Durkheim E. O suicídio. São Paulo: Martin Claret; 2008.358 p.

3. Werlang BSG, Borges VB, Fensterseifer L. Fatores de Risco ou Proteção para a Presença de ideaçcão suicida na adolescência. Rev Interam Psicol. 2005;39(2):259-66.

4. Organização Mundial da Saúde. Prevenção do suicídio: um manual para profissionais das equipes de saúde mental. Brasília: OMS; 2006.

5. Abasse MLF, Oliveira RC, Silva TC, Souza ER. Análise epidemiológica da morbimortalidade por suicídio entre adolescentes em Minas Gerais, Brasil. Ciênc Saúde Coletiva. mar-abr 2009;14(2):407-16.

6. Botega NJ. Suicídio: saindo da sombra em direção a um Plano Nacional de Prevenção. Rev Bras Psiquiatr. set-dez 2007; 29(1):7-8.

7. Ruengorn C, Sanichwankul k, Niwatananun W, Mahatnirunkul S, Pumpaisalchai W, Atumanond J. A riskscoring scheme for suicide attempts among patients with bipolar disorder in a Thai patient cohort. Psychol Res Behav Manage. 2012;(5):37-45.

8. Soloff PH, Chiappetta L. Prospective Predictors of Suicidal Behavior in BPD at 6 Year Follow-up. Am J Psychiatry 2012 May;169(5):484-90.

9. Pires MCC, Kurtinaitis LCL, Santos MSP, Passos MP, Sougey EB, Bastos OC Filho. Fatores de risco para tentativa de suicídio em idosos. Neurobiologia. out-dez 2009;72(4):21-8.

10. Ceará. Secretaria da Saúde do Estado. Doenças e agravos não transmissíveis - DANT no Ceará: situação epidemiológica, 1998 a 2004. Fortaleza: SESA; 2006.

11. Ministério da Saúde (BR). Relatório mundial de saúde 2003: Construindo o Futuro. Genebra: Organização Mundial de Saúde; 2003.

12. Lovisi GM, Santos AS, Legay L, Abelha L, Valencia E. Análise epidemiológica do suicídio no Brasil entre 1980 e 2006. Rev Bras Psiquiatr. 2009;31 Suppl 2:S86-93.
13. Regadas RP, Veras TN, Lins EB, Cavalcante LO, Aguiar JC, Davis M. Tentativas de suicídio por auto envenenamento: um estudo retrospectivo de 446 casos. Pesq Med Fortaleza. jan-dez 2000;3(1-4):50-3.

14. Avanci RC, Pedrão LJ, Costa ML Junior. Tentativa de suicídio na adolescência: considerações sobre a dificuldade de realização diagnóstica e a abordagem do profissional de enfermagem. SMAD, Rev. Eletrônica Saúde Mental Álcool Drog. (Ed. port.) [Internet]. 2005. [acesso 5 junho 2012];1(1). Disponível em: http:// www.revistasusp.sibi.usp.br/scielo.php?pid=S1806$69762005000100007 \&$ script=sci_arttext.

15. Souza ER, Minayo MCS, Cavalcante FG. O impacto do suicídio sobre a morbimortalidade da população de Itabira. Ciênc Saúde Coletiva. 2006;11 Supp 1:S1333-42.

16. Santos J. Suicídio em Mato Grosso do Sul, Brasil: fatores sociodemográficos. [Tese de Doutorado]. Rio de Janeiro (RJ): Escola de Saúde Pública da Fundação Oswaldo cruz; 2010. 65p.

17. Bezerra JG Filho, Werneck GL, Almeida RLF, Oliveira MIV, Magalhães FB. Estudo ecológico sobre os possíveis determinantes socioeconômicos, demográficos e fisiográficos do suicídio no Estado do Rio de Janeiro, Brasil, 1998-2002. Cad Saúde Pública. jan-mai 2012;28(5):833-44.

18. Pordeus AMJ, Cavalcanti LPG, Vieira LJES, Coriolano LS, Osório MM, Ponte MSR, et al. Tentativas e óbitos por suicídio no município de Independência, Ceará, Brasil. Ciênc Saúde Coletiva. nov-dez 2009;14(5):1731-40.

19. Denney JT, Rogers RG, Krueger PM, Wadsworth T. Adult Suicide Mortality in the United States: Marital Status, Family Size, ocioeconomic Status, and Differences by Sex. Soc Sci Q. 2009;90(5):1167-85.

20. Botega NJ, Werlang BSG, Cais CFS, Macedo MMK. Prevenção do comportamento suicida. Psico. set-dez 2006;37(3):213-20.

21. Costa J. Tentativa de Suicídio. [Dissertação de Mestrado]. Lisboa (Portugal): Faculdade de Ciências da Saúde. Universidade da Beira Interior. 2010. 78 p.

22. Chachamovich E, Stefanello S, Botega N, Turecki G. Quais são os recentes achados clínicos sobre a associação entre depressão e suicídio? Rev Bras Psiquiatr. 2009;31 Suppl 1:S18-25.

23. Meneghel SN, Victora CG, Faria NMX, Carvalho LA, Falk JW. Características epidemiológicas do suicídio no Rio Grande do Sul. Rev Saúde Pública. dez 2004;38 (6):804-10.

24. Bertolote JM, Mello-Santos C, Botega NJ. Detecção do risco de suicídio nos serviços de emergência Psiquiátrica. Rev Bras Psiquiatria. 2010;32 Suppl 2:S87-95.

25. Moreira CLRL, Brietzke E, Lafer B. General medical comorbidities in Brazilian outpatients with bipolar disorder type I. Rev Psiq Clín. 2011;38(6):227-30. 